\title{
New Adaptive Neural Network for Uncertain Nonlinear System with Disturbance
}

\author{
Thiem V. Pham ${ }^{1,2}$, Lai K. Lai ${ }^{1}$ \\ 1 Thai Nguyen University of Technology (TNUT), Viet Nam \\ ${ }^{2}$ CReSTIC, University of Reims Champagne-Ardenne (URCA), France
}

Correspondence: Thiem V. Pham, phuthiem@gmail.com

Communication: received 26 December 2016, revised 23 May 2017, accepted 10 July 2017

Online publication: 30 October 2017, Digital Object Identifier: 10.21553/rev-jec.151

The associate editor coordinating the review of this article and recommending it for publication was Prof. Tran Duc Tan.

\begin{abstract}
The paper deals with the problem of tracking control for a class of nonlinear systems in presence of the disturbances. The developed formation for the tracking control is taken into account as an adaptive neural network. The set of controller's parameter, which is a satisfy Hurwitz polynomial, is then updated by adaptive laws via a model reference system. In addition, the unknown nonlinear functions are estimated by radial basis functions neural network. The adaptive updated law based on radial basis functions neural network and a feed-forward correction is proposed to estimate both estimation errors of nonlinear functions and external disturbances, which is called lumped disturbances. The feed-forward correction term is calculated by the algebraic equation regarding the parameters of controller and the radial basis function. Moreover, this estimator (using estimating the lumped disturbance) is also used both in class of SISO nonlinear and MIMO nonlinear system. Thanks to Lyapunov's theory, asymptotic stability is established with the tracking errors converging to a neighborhood of the origin. Finally, two examples of coupled tank liquid system and an active magnetic bearing system, are presented to illustrate the our proposed methods.
\end{abstract}

Keywords- Radial Basis Function (RBF) Neural Network, Adaptive Scheme, Disturbance Estimation, Tracking Control, Nonlinear System, Liquid Level Systtem, Active Magnetic Bearing System.

\section{INTRODUCTION}

$\mathbf{T}$ RACKING control for uncertain nonlinear systems with unknown disturbances is a challenging problem [1] and [2]. To achieve good tracking under uncertainties, one often need to combine several methods in the control design such as adaption, feedforward, and high-gain, this paper is no exception. With the nonlinear system [1, 2] and [3] they use the exact feedback linearization approach to define a control law with assumption that the modeled object is clearly known and states are measured and available for feedback. If we do not know exactly parameters of plant, the linear parameterization of unknown dynamics poses serious obstacles in adopting adaptive control algorithms in practical applications, because it is difficult to fix the structure of the unknown nonlinearities. This fact has been the motivating factor behind the interest in on-line function approximators used in adaptive control which are artificial neural network and fuzzy logic system.

The nonlinear system with unknown input noise [2] and [4] has an uncertain parameter vector or unknown functions. Four trends will appear, the first trend consists in introducing a direct adaptive fuzzy control combined with sliding mode control [5] and [6] based on Lyapunov theory. The second trend is carried out in controller design based on universal approximation [7], [8] and [9], the indirect adaptive fuzzy control (IAFC) combined with sliding mode control (SMC) has attracted much attention. The combining the two achieving more superior performances such as overcoming some limitations of the traditional SMC. However, SMC suffers from a well-known problem chattering due to the high gain and high-speed switching control. The undesirable chattering may excite previously unmodelled system dynamics and damage actuators, resulting in unpredictable stability. The third trend, using ISS stabilization in [10] and [11] combined principles of certainty equivalence, a tracking errors smoothly converge to the arbitrarily sufficient small neighborhood of the origin. The last trend, employing an $H_{\infty}$ control with fuzzy approximation [12] and [13], consists in synthesizing an adaptation law, and a control law calculated from Riccati equation to attenuate the effect of both the approximation error and external disturbances. But this method requires the determination of the weighting matrix by the designer and find a trade off between the initial values of the control signal and the attenuation level.

In this work, artificial neural network is employed. From the point of view of possible drawbacks compared to the MPL (Multilayer Perceptron) which adjust only the connection weight matrix. Meanwhile, RBF (Radial Basis Function) neural networks [2] are characterized by two sets of adjustable parameters: the centers of the radial basis and the connection matrix. however, the RBF's output depends linearly on the connection weights and thus the training becomes a 
linear optimization problem. Therefore, in this paper we consider RBF in designing the adaptive controller.

Furthermore, instead of using sliding mode control as [13] and [14] we will consider the unknown input noise and error structure which is generated by general approximators can be lumped into a disturbance term. The lumped disturbance signal is estimated based on a RBF neural network combined with a feed-forward correction term. In addition, the parameters of controllers in [5], [9] and [15] are chosen such as they are coefficients of hurwitz polynomial that mean these controllers ensure only the stable closed loop system. On the other hand, these papers do not focus on the performance of closed loop system such as: the settling time, overshoot and error the output and reference signal. Thus, in this paper we concentrate to these problems.

The remainder of this paper is organized as follows. In Section 2, we introduce the optimal tracking control with based on adaptive RBF neural network and adaptive parameters of controller. Section 3 then provides the new adaptive RBF neural network combined with disturbance estimation, and extension adaptive neural network combined with disturbance estimation for MIMO nonlinear system could be represented in Section 4. Simulation results are given in Section 5 and Section 6 concludes this work.

\section{OPTIMAL TRACKING CONTROL BASED ON ADAPTIVE RBF NEURAL NETWORK (TC-RBF)}

As mentioned above, the RBF neural network can be considered as a two layers network with one hidden layer. The output depends linearly on the connection weights, the training is thus a linear optimization problem [2].

More explicitly, the RBF neural network performs the transformation: $b: R^{n} \rightarrow R$ with

$$
b(\boldsymbol{x})=\sum_{j=1}^{m} \Xi_{j}(\boldsymbol{x}) \boldsymbol{\Theta}_{j}=\boldsymbol{\Theta}^{T} \boldsymbol{\Xi}(\boldsymbol{x}),
$$

in which $\Xi_{j}(x)=\phi\left(\left\|x-c_{j}\right\|_{2}\right), x$ is the input vector, $\phi$ is a nonlinear function, called radial basis function, $\boldsymbol{\Theta}_{j}$ are connection weights between the hidden layer and the output layer, $c_{j}$ are centers of the basis function, $m$ is the number of basis function. The most common basis function is the Gaussian function

$$
\phi(x)=\exp \left(\frac{-r^{2}}{2 \sigma^{2}}\right)
$$

where $r=\left\|x-c_{j}\right\|_{2}, \sigma$ is an associated constant to the function $\phi(x)$ and represents the width of Gaussian function. Although the RBF neural network is usually considered linear parameterized, by adjusting the centers and the widths this type of neural network structure becomes nonlinearly parameterized. In this work, the centers of the basis function are chosen by constant and the connection weights are online adjusted.

\subsection{Tracking control based on RBF neural network}

Consider the SISO nonlinear system with disturbance $d(x, t)$ given as:

$$
\left\{\begin{array}{l}
y^{(n)}=f(\boldsymbol{x})+h(\boldsymbol{x}) u+d(\boldsymbol{x}, t) ;\|d(\boldsymbol{x}, t)\| \leq \rho \\
\boldsymbol{x}=\left(x_{1} \ldots x_{n}\right)^{T} \in \boldsymbol{R}^{n} ; u \in \boldsymbol{R}^{n \times m} ; f(\boldsymbol{x}) ; h(\boldsymbol{x}) \in C^{\infty},
\end{array}\right.
$$

where $f(x) \in \boldsymbol{R}^{n}$ and $h(x) \in \boldsymbol{R}^{n}$ are supposed to be unknown (uncertain) but with a limited bound, $u \in R^{n}$ is the input, $y \in \boldsymbol{R}^{n}$ is the outputs of the system, $x$ is the state vector, which is assumed to be available for measurement and $d \in \boldsymbol{R}^{n}$ represents the unknown but bounded and smooth external disturbances (load, white noise...).

Assumption 1:

$$
\|d(x, t)\| \leq \rho \text { and } d(x, t) \in C^{\infty}
$$

The objective is to design a feedback control law to drive the system output $y$ tracking a reference output $y_{m}$. With the assumption that all the state of the system are measurable and available for feedback.

In case of $d(x, t)=0$, the mentioned control target can be met by applying the state feedback controller:

$$
u_{c}=h^{-1}(x)\left(-f(x)+y_{m}^{(n)}+\sum_{i=1}^{n-1} k_{i} e^{(i)}\right) ; e(t)=y_{m}-y
$$

where $k_{i}, i=1,2, . . n-1$ are coefficients of hurwitz polynomial

$$
p(s)=k_{1}+k_{2} s+\ldots+k_{n-1} s^{n-2}+s^{n-1}
$$

It is not difficult to prove that the closed loop system with the control law (5) to be stable and the output error asymptotically approaches to zero.

Assuming that the functions $f(\boldsymbol{x})$ and $h(\boldsymbol{x})$ describing the system dynamics are unknown; the ideal control law cannot be implemented. Thus, $f(\boldsymbol{x})$ and $h(\boldsymbol{x})$ are approximated by a universal approximator of the following form:

$$
\hat{f}(\boldsymbol{x})=\boldsymbol{\Theta}_{f}^{T} \boldsymbol{\Xi}_{f}(\boldsymbol{x}) ; \hat{h}(\boldsymbol{x})=\boldsymbol{\Theta}_{h}^{T} \boldsymbol{\Xi}_{h}(\boldsymbol{x})
$$

where $\boldsymbol{\Xi}_{f}(\boldsymbol{x}), \boldsymbol{\Xi}_{h}(\boldsymbol{x})$ are the basic function vectors and $\boldsymbol{\Theta}_{f}^{T}, \Theta_{g}^{T}$ the parameters vectors, respectively.

The parameter vector $\Theta^{T}$ is updated online so that the approximation errors between $f(x), h(x)$ and $\widehat{f}(\boldsymbol{x}), \hat{h}(\boldsymbol{x})$ are minimal. Define the optimal parameters vector as:

$$
\begin{aligned}
& \boldsymbol{\Theta}_{f}^{*}=\underset{\boldsymbol{\Theta}_{f}}{\arg \min }\left(\sup _{x} \mid \boldsymbol{\Theta}_{f}^{T} \boldsymbol{\Xi}_{f}(\boldsymbol{x})-f(\boldsymbol{x})\right) \\
& \boldsymbol{\Theta}_{h}^{*}=\underset{\boldsymbol{\Theta}_{h}}{\arg \min }\left(\sup _{x} \mid \boldsymbol{\Theta}_{h}^{T} \boldsymbol{\Xi}_{h}(\boldsymbol{x})-h(\boldsymbol{x})\right)
\end{aligned}
$$

if $\boldsymbol{\Theta}_{f} \rightarrow \boldsymbol{\Theta}_{f}^{*} ; \boldsymbol{\Theta}_{h} \rightarrow \boldsymbol{\Theta}_{h}^{*}$ then $\hat{f}(\boldsymbol{x}) \rightarrow f(\boldsymbol{x}), \hat{h}(\boldsymbol{x}) \rightarrow h(\boldsymbol{x})$ that means the RBF neural network (8) can approximate smooth nonlinear functions with the arbitrary small error. In general cases, $f(\boldsymbol{x}), h(\boldsymbol{x})$ are not identical to $f(\boldsymbol{x}), h(\boldsymbol{x})$. Let $\delta_{f}(\boldsymbol{x}), \delta_{h}(\boldsymbol{x})$ are the structure error:

$$
\begin{aligned}
& f(\boldsymbol{x})=\boldsymbol{\Theta}_{f}^{* T} \boldsymbol{\Xi}_{f}(\boldsymbol{x})+\delta_{f}(\boldsymbol{x}) ; \\
& h(\boldsymbol{x})=\boldsymbol{\Theta}_{h}^{* T} \boldsymbol{\Xi}_{h}(\boldsymbol{x})+\delta_{h}(\boldsymbol{x})
\end{aligned}
$$




\subsection{Optimal Tracking Control}

With the estimated function $f(x)$ and $h(x)$ in (7). The closed loop system including state feedback controller (21) and uncertain nonlinear system with disturbance (3) can be expressed

$$
\begin{aligned}
& e^{n}=-k_{1} e-k_{2} \dot{e}-k_{3} \ddot{e}-\ldots-k_{n-1} e^{n-1}- \\
& -\delta_{f}(x)-\delta_{h}(x) u(t)
\end{aligned}
$$

in which, the parameters $k_{1} ; k_{2} ; \ldots ; k_{n-1}$ of the state feedback controller are chosen in (6). They only guarantee the closed loop system stable but, the tracking quality of closed loop is not considered.

On the other hand, it is clear that the performance of closed loop system such as the settling time, overshoot and error between the output signal and reference depending on the parameters $k_{1}, k_{2}, . ., k_{n}$. For example, the roots of the second order linear ordinary differential equation with $n=2 ; \Delta(\boldsymbol{x})=\delta_{f}(\boldsymbol{x})+\delta_{h}(\boldsymbol{x}) u(t)$

$$
\begin{aligned}
e_{0}(t)= & k_{1} \exp \left(0.5 t \sqrt{-k_{2}^{2}+4 k_{1}}+k_{2}\right)+ \\
& +k_{2} \exp \left(0.5 t \sqrt{k_{2}^{2}+4 k_{1}}\right. \\
e(t)= & e_{0}(t)-k_{1}^{-1} \sup _{x}|\Delta(x)|
\end{aligned}
$$

Therefore, to improve tracking performance of system, (the error between reference trajectory and output trajectory, the settling time), we need to find the optimal parameters or the adaptive parameters. In this paper, we propose an adaptive law for the parameters $k_{1} ; k_{2} ; \ldots ; k_{n-1}$.

The controller (5) can be rewritten as

$$
u_{c}=\hat{h}^{-1}(\boldsymbol{x})\left(-\hat{f}(\boldsymbol{x})+y_{m}^{(n)}+\boldsymbol{\Theta}_{k}^{T} \boldsymbol{e}\right)
$$

where $\boldsymbol{\Theta}_{k}^{T}=\left(k_{1}, k_{2}, \ldots, k_{n-1}\right) ; \boldsymbol{e}=\left(e \dot{e}, \ldots, e^{(n-1)}\right)^{T}$. The $\boldsymbol{\Theta}_{k}^{T}$ will be updated by the adaptive law based on reference system in theorem 1. Assumption 2: The function $h(\boldsymbol{x}), \hat{h}(\boldsymbol{x})$ are bounded

$$
0<h_{l o} \leq h(x), \hat{h}(x) \leq h_{u p}<\infty
$$

Theorem 1. Consider the nonlinear system (3) with the assumptions (4) and (13). If we choose the controller (12) in which the $\boldsymbol{\Theta}_{h}^{T}, \mathbf{\Theta}_{f}^{T}$ updated by the adaptive law

$$
\dot{\Theta}_{f}=-Q_{f}^{-1} \Xi_{f}(x) \varepsilon ; \dot{\Theta}_{h}=-Q_{h}^{-1} \Xi_{h}(x) \varepsilon u_{c}
$$

and the parameters of controller are updated by

$$
\begin{aligned}
& k_{1}(t)=k_{10}+\frac{1}{q_{k 11}} \int_{0}^{t}\left(y_{m}-y\right) \varepsilon d t \\
& k_{2}(t)=k_{20}+\frac{1}{q_{k 22}} \int_{0}^{t}\left(\dot{y}_{m}-\dot{y}\right) \varepsilon d t \\
& \vdots \\
& k_{n-1}(t)=k_{(n-1) 0}+a_{n-1} \int_{0}^{t}\left(y_{m}^{(n-1)}-y^{(n-1)}\right) \varepsilon d t
\end{aligned}
$$

where $a_{n-1}=\frac{1}{q_{k(n-1)(n-1)}}$. Then we can have $\lim _{t \rightarrow \infty} e(t) \rightarrow 0$ and the closed loop system is stable.
Proof: The nth derivative of the output error between output and reference is expressed:

$$
\begin{aligned}
& y^{(n)}=-(\hat{f}(\boldsymbol{x})-f(\boldsymbol{x}))-(\hat{h}(\boldsymbol{x})-h(\boldsymbol{x})) u_{c}+y_{m}^{(n)}+\boldsymbol{\Theta}_{k}^{T} e \\
& \dot{x}(n-1) m-\dot{x}_{n-1}=\left(\boldsymbol{\Theta}_{f}^{T} \boldsymbol{\Xi}_{f}(\boldsymbol{x})-\boldsymbol{\Theta}_{f}^{* T} \boldsymbol{\Xi}_{f}(\boldsymbol{x})-\delta_{f}(\boldsymbol{x})\right)- \\
& \boldsymbol{\Theta}_{k}^{T} e+\left(\boldsymbol{\Theta}_{h}^{T} \boldsymbol{\Xi}_{h}(\boldsymbol{x})-\boldsymbol{\Theta}_{h}^{* T} \boldsymbol{\Xi}_{h}(\boldsymbol{x})-\delta_{h}(\boldsymbol{x})\right) u_{c} \\
& \dot{\varepsilon}=\left(\boldsymbol{\Theta}_{f}^{T}-\boldsymbol{\Theta}_{f}^{* T}\right) \boldsymbol{\Xi}_{f}(\boldsymbol{x})+\left(\boldsymbol{\Theta}_{h}^{T}-\boldsymbol{\Theta}_{h}^{* T}\right) \boldsymbol{\Xi}_{h}(\boldsymbol{x}) u_{c}- \\
& -\boldsymbol{\Theta}_{k}^{T} \boldsymbol{e}-\left[\delta_{f}(\boldsymbol{x})+\delta_{h}(\boldsymbol{x}) u_{c}\right] \\
& \dot{\varepsilon}=\tilde{\boldsymbol{\Theta}}_{f}^{T} \boldsymbol{\Xi}_{f}(\boldsymbol{x})+\tilde{\boldsymbol{\Theta}}_{h}^{T} \boldsymbol{\Xi}_{h}(\boldsymbol{x}) u_{c}-\Delta(\boldsymbol{x})-\boldsymbol{\Theta}_{k}^{T} e \\
& \tilde{\boldsymbol{\Theta}}_{f}^{T}=\left(\boldsymbol{\Theta}_{f}^{T}-\boldsymbol{\Theta}_{f}^{* T}\right) ; \tilde{\boldsymbol{\Theta}}_{h}^{T}=\left(\boldsymbol{\Theta}_{h}^{T}-\boldsymbol{\Theta}_{h}^{* T}\right) ;
\end{aligned}
$$

Consider the Lyapunov candidate function:

$$
\begin{aligned}
& V=\frac{1}{2} \varepsilon^{2}+\frac{1}{2} \tilde{\mathbf{\Theta}}_{f}^{T} \boldsymbol{Q}_{f} \tilde{\boldsymbol{\Theta}}_{f}^{T}+\frac{1}{2} \tilde{\boldsymbol{\Theta}}_{h}^{T} \boldsymbol{Q}_{h} \tilde{\boldsymbol{\Theta}}_{h}^{T}+\frac{1}{2} \mathbf{\Theta}_{k}^{T} \boldsymbol{Q}_{k} \mathbf{\Theta}_{k} ; \\
& \boldsymbol{Q}_{f}>0 ; \boldsymbol{Q}_{g}>0 ; \boldsymbol{Q}_{k}>0
\end{aligned}
$$

where $Q_{i} \in \boldsymbol{R}^{d \times d}\left(d=\operatorname{dim} \boldsymbol{\Theta}_{i}\right)$ is a positive definite matrix. Take the derivative of $V$ with respect to time and notice that $\tilde{\Theta}_{i}=\dot{\Theta}_{i}$, we have:

$$
\begin{aligned}
& \dot{V}=\varepsilon \dot{\varepsilon}+\tilde{\boldsymbol{\Theta}}_{f}^{T} \boldsymbol{Q}_{f} \dot{\boldsymbol{\Theta}}_{f}+\tilde{\mathbf{\Theta}}_{h}^{T} \boldsymbol{Q}_{h} \dot{\boldsymbol{\Theta}}_{h}+\boldsymbol{\Theta}_{k}^{T} \boldsymbol{Q}_{k} \dot{\boldsymbol{\Theta}}_{k} \\
& \dot{V}=\tilde{\boldsymbol{\Theta}}_{f}^{T}\left(\varepsilon \boldsymbol{\Xi}_{f}(x)+\boldsymbol{Q}_{f} \dot{\boldsymbol{\Theta}}_{f}\right)+\tilde{\boldsymbol{\Theta}}_{h}^{T}\left(\varepsilon \boldsymbol{\Xi}_{h}(\boldsymbol{x}) u_{c}+\boldsymbol{Q}_{h} \dot{\boldsymbol{\Theta}}_{h}\right)+ \\
& +\boldsymbol{\Theta}_{k}^{T}\left(\boldsymbol{Q}_{k} \dot{\boldsymbol{\Theta}}_{k}-\varepsilon e\right)-\varepsilon \Delta(\boldsymbol{x})
\end{aligned}
$$

Chose the parameter update law to cancel the parameter error as follow:

$$
\begin{gathered}
\varepsilon \Xi_{f}(\boldsymbol{x})+\boldsymbol{Q}_{f} \dot{\boldsymbol{\Theta}}_{f}=0 \Rightarrow \dot{\boldsymbol{\Theta}}_{f}=-\boldsymbol{Q}_{f}^{-1} \boldsymbol{\Xi}_{f}(\boldsymbol{x}) \varepsilon \\
\varepsilon \Xi_{h}(\boldsymbol{x}) u_{c}+\boldsymbol{Q}_{h} \dot{\boldsymbol{\Theta}}_{h}=0 \Rightarrow \dot{\boldsymbol{\Theta}}_{h}=-\boldsymbol{Q}_{h}^{-1} \boldsymbol{\Xi}_{h}(\boldsymbol{x}) \varepsilon u_{c} \\
\boldsymbol{Q}_{k} \dot{\boldsymbol{\Theta}}_{k}-\varepsilon e=0 \Rightarrow \dot{\boldsymbol{\Theta}}_{k}=\boldsymbol{Q}_{k}^{-1} \varepsilon e
\end{gathered}
$$

where a weighting matrix $Q_{k}$ is given as:

$$
\begin{aligned}
& Q_{k}=\left(\begin{array}{ccc}
q_{k 11} & \cdots & 0 \\
\vdots & \ddots & \vdots \\
0 & \cdots & q_{k(n-1)(n-1)}
\end{array}\right) ; \dot{\boldsymbol{\Theta}}_{k}=Q_{k}^{-1} \mathcal{E} e \quad(18) \\
& k_{1}(t)=k_{1}(0)+\frac{1}{q_{k 11}} \int\left(y_{m}-y\right) \varepsilon d t \\
& \vdots \\
& k_{n-1}(t)=k_{n-1}(0)+\frac{1}{q_{k(n-1)(n-1)}} \int\left(y_{m}^{(n-1)}-y^{(n-1)}\right) \varepsilon d t
\end{aligned}
$$

and $k_{1}(0), k_{2}(0), \ldots, k_{n-1}(0)$ are chosen in (6) to guarantee the stability of the closed loop system. Finally, we have:

$$
\dot{V}=-\varepsilon \Delta(x) \leq-|\varepsilon \Delta(x)| \leq 0
$$

Since $V$ is quadratic function and $\dot{V} \leq 0$, the control system is proven to be stable.

Remark 1. Following (11), when there is an error structures, then $k_{1}^{-1} \sup _{x, t=t}|\Delta(x, t)|$ will be reduced dramatically by automatic adjusted parameters $k_{1}$ this means that the adaptive parameters of controller contribute a decrease affection of error structures. 


\section{NEW ADAPTIVE RBF NEURAL NETWORK AND DISTURBANCE ESTIMATION (NA-RBF-DE)}

In this section, we consider an affection of disturbance on system $d(\boldsymbol{x}, t) \neq 0$. The nonlinear system (3) can be expressed:

$$
\begin{aligned}
y^{(n)} & =\boldsymbol{\Theta}_{F}^{* T} \boldsymbol{\Xi}_{F}(\boldsymbol{x})+\delta_{F}(\boldsymbol{x})+ \\
& +\left[\boldsymbol{\Theta}_{H}^{* *} \boldsymbol{\Xi}_{H}(\boldsymbol{x})+\delta_{H}(\boldsymbol{x})\right] u+d(\boldsymbol{x}, t) \\
& =\boldsymbol{\Theta}_{F}^{* T} \boldsymbol{\Xi}_{F}(\boldsymbol{x})+\boldsymbol{\Theta}_{H}^{* T} \boldsymbol{\Xi}_{H}(\boldsymbol{x}) u+\eta(\boldsymbol{x}, t) \\
\eta(\boldsymbol{x}, t) & =d(\boldsymbol{x}, t)+\delta_{F}(\boldsymbol{x})+\delta_{H}(\boldsymbol{x}) u_{c}
\end{aligned}
$$

in which the $\eta(x, t)$ is called lumped disturbance.

If $\eta(x, t) \neq 0$, the controller (12) could only drive the system output to a neighborhood of the desired trajectory. We will introduce another control law combined disturbance estimation with adaptive RBF neural network. In which, $\eta(x, t)$ is estimated by the adaptive RBF neural network $\hat{\eta}(\boldsymbol{x}, t)=\boldsymbol{\Theta}_{\eta}^{T} \boldsymbol{\Xi}_{\eta}(\boldsymbol{x})$. The state feedback controller is selected as

$$
\begin{aligned}
& u=u_{c}+u_{d} \\
& u_{d}=-\hat{h}^{-1}(\boldsymbol{x}) \hat{\eta}(x, t) \\
& u_{c}=\hat{h}^{-1}(\boldsymbol{x})\left(-\hat{f}(\boldsymbol{x})+y_{m}^{(n)}+\boldsymbol{\Theta}_{k} \boldsymbol{e}\right)
\end{aligned}
$$

Theorem 2. Consider the nonlinear system (3) with the assumptions (4) and (13). If we choose the controller (21) in which the $\mathbf{\Theta}_{h}^{T}, \mathbf{\Theta}_{f}^{T}$ updated by the adaptive law

$$
\dot{\Theta}_{f}=-Q_{f}^{-1} \Xi_{f}(x) \varepsilon ; \dot{\Theta}_{h}=-Q_{h}^{-1} \Xi_{h}(x) \varepsilon u_{c}
$$

the parametters of controller are updated

$$
\begin{aligned}
& k_{1}(t)=k_{10}+\frac{1}{q_{k 11}} \int_{0}^{t}\left(y_{m}-y\right) \varepsilon d t \\
& k_{2}(t)=k_{20}+\frac{1}{q_{k 22}} \int_{0}^{t}\left(\dot{y}_{m}-\dot{y}\right) \varepsilon d t \\
& \vdots \\
& k_{n-1}(t)=k_{(n-1) 0}+a_{n-1} \int_{0}^{t}\left(y_{m}^{(n-1)}-y^{(n-1)}\right) \varepsilon d t
\end{aligned}
$$

and the disturbance estimation is obtained form

$$
\hat{\eta}(x, t)=-\int_{0}^{t} \boldsymbol{\Xi}_{\eta}^{T}(x)\left(Q_{\eta}^{-1}\right)^{T} \varepsilon d t \Xi_{\eta}(x)-\gamma e(t)
$$

where $\gamma$ is root of $\gamma A_{d}+\gamma B_{d} \gamma+\Xi_{\eta}^{T}(x) Q_{\eta}^{-1} \Xi_{\eta}(x) a_{d}=0$. Then we can have $\lim _{t \rightarrow \infty} e(t) \rightarrow 0, \lim _{t \rightarrow \infty}(\hat{\eta}(x, t)-\eta(x, t)) \rightarrow$ 0 and the closed loop system is stable.

Proof: There are two parts in our proof. First, the stability of closed loop system will be given by Lyapunov function. After that, an explaining to a feedforward correction $\Gamma$ is obtained by (23) and a proof of convergence of estimated disturbance error. First of all, a dynamic of error will be expressed

$$
\begin{aligned}
& y^{(n)}=f(\boldsymbol{x})+h(\boldsymbol{x})\left(u_{c}+u_{d}\right)+d(\boldsymbol{x}, t) \\
& =-(\hat{f}(\boldsymbol{x})-f(\boldsymbol{x}))-(\hat{h}(\boldsymbol{x})-h(\boldsymbol{x})) u_{c} \\
& +\hat{f}(\boldsymbol{x})+\hat{h}(\boldsymbol{x}) u_{c}+h(\boldsymbol{x}) u_{d}+d(\boldsymbol{x}, t) \\
& \dot{\varepsilon}=\left(\boldsymbol{\Theta}_{f}^{T} \boldsymbol{\Xi}_{f}(\boldsymbol{x})-\boldsymbol{\Theta}_{f}^{* T} \boldsymbol{\Xi}_{f}(\boldsymbol{x})-\delta_{f}(\boldsymbol{x})\right)-h(\boldsymbol{x}) u_{d}+ \\
& +\left(\boldsymbol{\Theta}_{h}^{T} \boldsymbol{\Xi}_{h}(\boldsymbol{x})-\boldsymbol{\Theta}_{h}^{* T} \boldsymbol{\Xi}_{h}(\boldsymbol{x})-\delta_{h}(\boldsymbol{x})\right) u_{c}-\boldsymbol{\Theta}_{k}^{T} \boldsymbol{e}-d(\boldsymbol{x}, t) \\
& \dot{\varepsilon}=\left(\boldsymbol{\Theta}_{f}^{T}-\boldsymbol{\Theta}_{f}^{* T}\right) \boldsymbol{\Xi}_{f}(x)+\left(\boldsymbol{\Theta}_{h}^{T}-\boldsymbol{\Theta}_{h}^{* T}\right) \boldsymbol{\Xi}_{h}(\boldsymbol{x}) u_{c}- \\
& -\left[\delta_{f}(\boldsymbol{x})+\delta_{h}(\boldsymbol{x}) u_{c}+d(\boldsymbol{x}, t)\right]-h(\boldsymbol{x}) u_{d}-\boldsymbol{\Theta}_{k}^{T} \boldsymbol{e} \\
& =\tilde{\mathbf{\Theta}}_{f}^{T} \boldsymbol{\Xi}_{f}(\boldsymbol{x})+\tilde{\boldsymbol{\Theta}}_{h}^{T} \boldsymbol{\Xi}_{h}(\boldsymbol{x}) u_{c}-\eta(\boldsymbol{x}, t)-h(\boldsymbol{x}) u_{d}-\boldsymbol{\Theta}_{k}^{T} \boldsymbol{e} \\
& =\tilde{\boldsymbol{\Theta}}_{f}^{T} \boldsymbol{\Xi}_{f}(\boldsymbol{x})+\tilde{\boldsymbol{\Theta}}_{h}^{T} \boldsymbol{\Xi}_{h}(\boldsymbol{x}) u_{c}+(\hat{\eta}(\boldsymbol{x}, t)-\eta(\boldsymbol{x}, t))- \\
& -\hat{\eta}(\boldsymbol{x}, t)-h(\boldsymbol{x}) u_{d}-\boldsymbol{\Theta}_{k}^{T} \boldsymbol{e} \\
& =\tilde{\boldsymbol{\Theta}}_{f}^{T} \boldsymbol{\Xi}_{f}(x)+\tilde{\boldsymbol{\Theta}}_{h}^{T} \boldsymbol{\Xi}_{h}(\boldsymbol{x}) u_{c}+\tilde{\boldsymbol{\Theta}}_{\eta}^{T} \boldsymbol{\Xi}_{\eta}(\boldsymbol{x})-\hat{\eta}(\boldsymbol{x}, t)- \\
& -h(\boldsymbol{x}) u_{d}-\boldsymbol{\Theta}_{k}^{T} \boldsymbol{e}
\end{aligned}
$$

in which $\tilde{\boldsymbol{\Theta}}_{f}^{T}=\left(\boldsymbol{\Theta}_{f}^{T}-\boldsymbol{\Theta}_{f}^{* T}\right) ; \tilde{\Theta}_{h}^{T}=\left(\boldsymbol{\Theta}_{h}^{T}-\Theta_{h}^{* T}\right) ; \tilde{\Theta}_{\eta}^{T}=$ $\left(\boldsymbol{\Theta}_{\eta}^{T}-\boldsymbol{\Theta}_{\eta}^{* T}\right)$

Consider the Lyapunov candidate function:

$$
\begin{aligned}
& V=\frac{1}{2} \varepsilon^{2}+\frac{1}{2} \tilde{\boldsymbol{\Theta}}_{f} \boldsymbol{Q}_{f} \tilde{\boldsymbol{\Theta}}_{f}^{T}+\frac{1}{2} \tilde{\mathbf{\Theta}}_{h} \boldsymbol{Q}_{h} \tilde{\boldsymbol{\Theta}}_{h}^{T}+ \\
& +\frac{1}{2} \tilde{\boldsymbol{\Theta}}_{\eta} \boldsymbol{Q}_{\eta} \tilde{\boldsymbol{\Theta}}_{\eta}^{T}+\frac{1}{2} \tilde{\boldsymbol{\Theta}}_{k} \boldsymbol{Q}_{k} \tilde{\boldsymbol{\Theta}}_{k}^{T}
\end{aligned}
$$

Take the derivative of $V$ with respect to time and notice that $\dot{\tilde{\Theta}}_{i}=\dot{\Theta}_{i}$, we have:

$$
\begin{aligned}
& \dot{V}=\varepsilon \dot{\varepsilon}+\tilde{\boldsymbol{\Theta}}_{f}^{T} \boldsymbol{Q}_{f} \dot{\boldsymbol{\Theta}}_{f}+\tilde{\boldsymbol{\Theta}}_{h}^{T} \boldsymbol{Q}_{h} \dot{\boldsymbol{\Theta}}_{h}+\tilde{\boldsymbol{\Theta}}_{\eta}^{T} \boldsymbol{Q}_{\eta} \dot{\boldsymbol{\Theta}}_{\eta}+\tilde{\boldsymbol{\Theta}}_{k}^{T} \boldsymbol{Q}_{k} \dot{\boldsymbol{\Theta}}_{k} \\
& \dot{V}=\varepsilon\left(\tilde{\boldsymbol{\Theta}}_{f}^{T} \boldsymbol{\Xi}_{f}(\boldsymbol{x})+\tilde{\boldsymbol{\Theta}}_{h}^{T} \boldsymbol{\Xi}_{h}(\boldsymbol{x}) u_{c}+\tilde{\boldsymbol{\Theta}}_{\eta}^{T} \boldsymbol{\Xi}_{\eta}(\boldsymbol{x})-\hat{\eta}(\boldsymbol{x}, t)-\right. \\
& \left.-h(\boldsymbol{x}) u_{d}-\boldsymbol{\Theta}_{k}^{T} \boldsymbol{e}\right)+\tilde{\boldsymbol{\Theta}}_{f}^{T} Q_{f} \dot{\boldsymbol{\Theta}}_{f}+ \\
& +\tilde{\boldsymbol{\Theta}}_{h}^{T} \boldsymbol{Q}_{h} \dot{\boldsymbol{\Theta}}_{h}+\tilde{\boldsymbol{\Theta}}_{\eta}^{T} \boldsymbol{Q}_{\eta} \dot{\boldsymbol{\Theta}}_{\eta}+\tilde{\boldsymbol{\Theta}}_{k}^{T} \boldsymbol{Q}_{k} \dot{\boldsymbol{\Theta}}_{k} \\
& =\tilde{\boldsymbol{\Theta}}_{f}^{T}\left(\varepsilon \boldsymbol{\Xi}_{f}(\boldsymbol{x})+\boldsymbol{Q}_{f} \dot{\boldsymbol{\Theta}}_{f}\right)+\tilde{\boldsymbol{\Theta}}_{h}^{T}\left(\varepsilon \boldsymbol{\Xi}_{h}(\boldsymbol{x}) u_{c}+Q_{h} \dot{\boldsymbol{\Theta}}_{h}\right)+ \\
& +\tilde{\boldsymbol{\Theta}}_{n}^{T}\left(\varepsilon \boldsymbol{\Xi}_{n}(\boldsymbol{x})+\boldsymbol{Q}_{n} \dot{\boldsymbol{\Theta}}_{n}\right)+\boldsymbol{\Theta}_{k}^{T}\left(\boldsymbol{Q}_{k} \dot{\boldsymbol{\Theta}}_{k}-\varepsilon \boldsymbol{e}\right) \\
& -\varepsilon \hat{\eta}(\boldsymbol{x}, t)-\varepsilon h(\boldsymbol{x}) u_{d}
\end{aligned}
$$

Choose the parameter update law to cancel the parameter error as follow

$$
\begin{gathered}
\varepsilon \Xi_{f}(\boldsymbol{x})+\boldsymbol{Q}_{f} \dot{\boldsymbol{\Theta}}_{f}=0 \Rightarrow \dot{\boldsymbol{\Theta}}_{f}=-\boldsymbol{Q}_{f}^{-1} \boldsymbol{\Xi}_{f}(\boldsymbol{x}) \mathcal{\varepsilon} \\
\varepsilon \boldsymbol{\Xi}_{h}(\boldsymbol{x}) u_{c}+\boldsymbol{Q}_{h} \dot{\boldsymbol{\Theta}}_{h}=0 \Rightarrow \dot{\boldsymbol{\Theta}}_{h}=-\boldsymbol{Q}_{h}^{-1} \boldsymbol{\Xi}_{h}(\boldsymbol{x}) \varepsilon u_{c} \\
\boldsymbol{Q}_{k} \dot{\boldsymbol{\Theta}}_{k}-\varepsilon \boldsymbol{e}=0 \Rightarrow \dot{\boldsymbol{\Theta}}_{k}=-\boldsymbol{Q}_{k}^{-1} \varepsilon \boldsymbol{e} \\
\varepsilon \boldsymbol{\Xi}_{\eta}(\boldsymbol{x})+\boldsymbol{Q}_{\eta} \dot{\boldsymbol{\Theta}}_{\eta}=0 \Rightarrow \dot{\boldsymbol{\Theta}}_{\eta}=-\boldsymbol{Q}_{\eta}^{-1} \Xi_{\eta}(\boldsymbol{x}) \varepsilon
\end{gathered}
$$

after that we have:

$$
\dot{V}=-\varepsilon \hat{\eta}(x, t)-\varepsilon h(x) u_{d}
$$

Substitute $u_{d}=-\hat{h}^{-1}(\boldsymbol{x}) \hat{\eta}(x, t)$ and assumption 2 for (28)

$$
\begin{aligned}
& \dot{V}=\varepsilon \hat{\eta}(x, t)\left(\frac{h(x)}{h u p}-1\right) \\
& \dot{V} \leq|\varepsilon|\left(\frac{h(x)}{h_{u p}}-1\right)\|\hat{\eta}(x, t)\| \leq 0
\end{aligned}
$$

Since $V$ is quadratic function and $\dot{V} \leq 0$, the control system is proven to be stable. It is clear that $V \in L^{\infty}$, which implies $\varepsilon \in L^{\infty}$ and $\tilde{\Theta}_{i} \in L^{\infty}$. from (30) we have $\dot{e} \in L^{\infty}$. Consequently, from the definition of and (6), we have $e^{(j)} \in L^{\infty}, j=1, . ., r-1$. Also, since $\int_{0}^{t} \dot{V} d t=V(t)-V(0)<\infty$ we have $e \in L^{2}$. Since $e \in L^{2}$, we have $\dot{e} \in L^{\infty}$. From the facts that $\dot{e} \in L^{\infty}$ and $e \in L^{\infty} \cap L^{2}$ according to Barbalat's Lemma [16], we have $\lim _{t \rightarrow \infty} e(t) \rightarrow 0$. 
With adaptive law (25) and (26), the derivative of surface error can be expressed

$$
e^{(n)}+\sum_{i=1}^{n-1} k_{i} e^{(i)}=-\eta(x, t)+\hat{\eta}(x, t)
$$

then, we have

$$
\begin{gathered}
e^{(n)}=-k_{1} e-k_{2} \dot{e}-\ldots-k_{n-1} e^{(n-1)}+(\hat{\eta}(x, t)-\eta(x, t)) \\
\dot{e}=A_{d} e+\boldsymbol{B}_{d} e_{\eta} ; e_{\eta}=\hat{\eta}(x, t)-\eta(x, t) \\
A_{d}=\left(\begin{array}{ccccc}
0 & 1 & 0 & \cdots & 0 \\
0 & 0 & 1 & \cdots & 0 \\
\vdots & \vdots & \vdots & \ddots & \vdots \\
0 & 0 & 0 & 0 & 1 \\
-k_{1} & -k_{2} & -k_{3} & \cdots & -k_{n-1}
\end{array}\right) \\
\boldsymbol{e}=\left(\begin{array}{c}
e \\
\dot{e} \\
\vdots \\
e^{(n-2)} \\
e^{(n-1)}
\end{array}\right) ; \boldsymbol{B}_{d}=\left(\begin{array}{c}
0 \\
0 \\
\vdots \\
0 \\
1
\end{array}\right)
\end{gathered}
$$

from (27), we assume the disturbance estimation as

$$
-\boldsymbol{\Xi}_{\eta}^{T}(\boldsymbol{x}) \boldsymbol{Q}_{\eta}^{-1} \boldsymbol{\Xi}_{\eta}(\boldsymbol{x}) s(e)=\dot{\boldsymbol{\eta}}_{\boldsymbol{b}}(\boldsymbol{x}, t)=\dot{e}_{\eta 0}
$$

if the disturbances are constant, $\hat{\eta}_{0}(x, t)$ becomes true. In case of variable disturbance, the disturbances estimation are updated following the equation:

$$
\hat{\eta}(x, t)=\hat{\eta}_{0}(x, t)-\gamma \boldsymbol{e}(t) ; \gamma=\left(\gamma_{1}, \gamma_{2}, \ldots, \gamma_{n}\right)
$$

where $\hat{\eta}_{0}(\boldsymbol{x}, t)$ is pre-correction, and $\hat{\eta}(\boldsymbol{x}, t)$ is the corrected estimation for time-varying disturbances with correction gain $\gamma$. from $\dot{\boldsymbol{e}}=\boldsymbol{A}_{d} \boldsymbol{e}+\boldsymbol{B}_{d} e_{\eta}, \boldsymbol{e}(t)$ is generated from a linear dynamics excited by $e_{\eta 0}(t)$. Intuitively, we can assume that

$$
e_{\eta 0}(t)=\gamma(t) e(t)
$$

where $\gamma$ is potentially time varying. Take the derivative of (34), we have

$$
\begin{aligned}
\dot{e}_{\eta 0}(t) & =\dot{\gamma}(t) \boldsymbol{e}(t)+\gamma(t) \dot{\boldsymbol{e}}(t) \\
\quad & =\dot{\gamma}(t) \boldsymbol{e}(t)+\gamma(t)\left(\boldsymbol{A}_{d} \boldsymbol{e}(t)+\boldsymbol{B}_{d} \boldsymbol{e}_{\eta}\right) \\
& =\dot{\gamma}(t) \boldsymbol{e}(t)+\gamma(t) \boldsymbol{A}_{d} \boldsymbol{e}(t)+\gamma(t) \boldsymbol{B}_{d} \gamma(t) \boldsymbol{e}(t)
\end{aligned}
$$

In addition, $\dot{e}_{\eta 0}=-\boldsymbol{\Xi}_{\eta}^{T}(\boldsymbol{x}) \boldsymbol{Q}_{\eta}^{-1} \boldsymbol{\Xi}_{\eta}(\boldsymbol{x}) \boldsymbol{a}_{d} \boldsymbol{e}$, where $\boldsymbol{a}_{d}$ be the last column of $A_{d}$.

$$
\begin{aligned}
& -\boldsymbol{\Xi}_{\eta}^{T}(\boldsymbol{x}) \boldsymbol{Q}_{\eta}^{-1} \boldsymbol{\Xi}_{\eta}(\boldsymbol{x}) \boldsymbol{a}_{d} \boldsymbol{e}= \\
& \dot{\gamma}(t) \boldsymbol{e}+\gamma(t) \boldsymbol{A}_{d} \boldsymbol{e}+\gamma(t) \boldsymbol{B}_{d} \gamma(t) \boldsymbol{e}(t) \\
& \dot{\gamma}(t)=-\left[\gamma(t) \boldsymbol{A}_{d}+\gamma(t) \boldsymbol{B}_{d} \gamma(t)+\boldsymbol{\Xi}_{\eta}^{T}(\boldsymbol{x}) \boldsymbol{Q}_{\eta}^{-1} \boldsymbol{\Xi}_{\eta}(\boldsymbol{x}) \boldsymbol{a}_{d}\right]
\end{aligned}
$$

The steady state can be used

$$
\gamma A_{d}+\gamma \boldsymbol{B}_{d} \gamma+\boldsymbol{\Xi}_{\eta}^{T}(x) \boldsymbol{Q}_{\eta}^{-1} \boldsymbol{\Xi}_{\eta}(\boldsymbol{x}) \boldsymbol{a}_{d}=0
$$

where $\boldsymbol{\Xi}_{\eta}(\boldsymbol{x}) ; \boldsymbol{x}=\left(y, \dot{y}, . . y^{(n)}\right)$. In steady state, $\boldsymbol{\Xi}_{\eta}(\boldsymbol{x})=$ $(1,0, . ., 0)$. from $\dot{\boldsymbol{e}}=\boldsymbol{A}_{d} \boldsymbol{e}+\boldsymbol{B}_{d} e_{\eta}, \boldsymbol{e}(t)$ and $\dot{\boldsymbol{e}} \in L^{\infty}$ we have $\lim _{t \rightarrow \infty} \boldsymbol{B}_{d} \boldsymbol{e}_{\eta}(t) \rightarrow 0$. If $\boldsymbol{B}_{d}$ has full column rank, we can conclude that $\lim _{t \rightarrow \infty} e_{\eta}(t) \rightarrow 0$.

Remark 2. In case of the disturbance $d(\boldsymbol{x}, t)$ is unbounded. this means that it dose not satisfy the assumption 1 (4), we will be completely converted into form assumption 1 such as $\sup _{0 \leq \tau \leq t}|\widehat{d}(x, \tau)-d(x, \tau)| \leq \rho$ by employing estimated algorithm based on adaptive RBF neural network as present.

Remark 3. In the equation (36), the $A_{d}$ matrix and $\boldsymbol{a}_{d}$ vector depend on the adaptive gains $\mathbf{\Theta}_{k}^{T}=\left(k_{1}, k_{2}, \ldots, k_{n-1}\right)$. Consequently, calculating the feed-forward correction term $\gamma$ is corrected online, then the performance of estimation disturbance error will be improved. There is an algorithm to calculate $\gamma$

Input:

- Initial value $k_{10}, k_{20}, \ldots, k_{(n-1) 0}$ which satisfied the equation (6).

- Compute $k_{1}, k_{2}, \ldots, k_{(n-1)}$ following (14).

- Setup $\boldsymbol{B}_{d}, \boldsymbol{\Xi}_{\eta}(\boldsymbol{x})=(1,0, . ., 0), \boldsymbol{Q}_{\eta}=q \boldsymbol{I}_{m \times n}, q>0$

\section{Output:} is abitrary value.

- Vector $\gamma=\left(\gamma_{1}, \gamma_{2}, \ldots, \gamma_{n}\right)$ is calculated from (36). and the general structure of new controller for nonlinear system in shown in Fig. 1.

\section{EXTENSION OF ADAPTIVE NEURAL AND DISTURBANCE ESTIMATION FOR MIMO NONLINEAR SYSTEM (NASC-DE)}

The results of Theorem 1 and 2 are extended for MIMO nonlinear system given as

$$
\left\{\begin{array}{l}
\dot{x}_{k}=x_{k+1} \quad 1 \leq k \leq n-1 \\
\dot{x}_{n}=\boldsymbol{F}(\boldsymbol{x})+\boldsymbol{H}(\boldsymbol{x}) \boldsymbol{u}+\boldsymbol{d}(\boldsymbol{x}, t) ; \quad\|\boldsymbol{d}(\boldsymbol{x}, t)\| \leq \boldsymbol{\rho} \\
\boldsymbol{y}=x_{1}
\end{array}\right.
$$

in which $x=\left(x_{1}, \ldots, x_{n}\right)^{T} \in \boldsymbol{R}^{m} ; \quad x_{1}=$ $\left(x_{11}, x_{12}, \ldots, x_{1 m}\right)^{T} \boldsymbol{u}=\left(u_{1}, u_{2}, \ldots, u_{n}\right)^{T} \in \boldsymbol{R}^{n}$ and $\boldsymbol{F}(\boldsymbol{x}) ; \boldsymbol{H}(\boldsymbol{x})=\left(h_{1}(\boldsymbol{x}), h_{2}(\boldsymbol{x}), \ldots, h_{n}(\boldsymbol{x})\right)^{T} \in C^{\infty} ; \boldsymbol{d}(\boldsymbol{x}, t)=$ $\left(d_{1}(\boldsymbol{x}), d_{2}(\boldsymbol{x}), \ldots, d_{n}(\boldsymbol{x})\right)^{T}$.

The $\boldsymbol{F}(\boldsymbol{x}) \in \boldsymbol{R}^{n}$ and $\boldsymbol{H}(\boldsymbol{x}) \in \boldsymbol{R}^{n \times n}$ are supposed to be unknown (uncertain) but with a limited bound, $\boldsymbol{u} \in \boldsymbol{R}^{n}$ are the inputs, $\boldsymbol{u} \in \boldsymbol{R}^{n}$ are the outputs of the system, $x$ is the state vector, which is assumed to be available for measurement and $\boldsymbol{d}(\boldsymbol{x}, t) \in \boldsymbol{R}^{n}$ represents the unknown but bounded and smooth external disturbances (load, white noise....). In order for the system to be controllable, we require that $\operatorname{det}(\boldsymbol{H}(\boldsymbol{x})) \neq 0$ for $\boldsymbol{x}$ in the operational field of the system. Thus, the above $n$ degree system is in the normal form.

Assumption 3:

$$
\|\boldsymbol{d}(x, t)\| \leq \rho \text { and } \boldsymbol{d}(x, t) \in C^{\infty}
$$

The MIMO RBF neural network can be expressed as three layers (see Fig. 4): $\mathrm{n}$ inputs; one hidden layer with $\mathrm{m}$ neural and $\mathrm{n}$ outputs. The output depends linearly on the connection weights. The MIMO RBF neural network can be performed such as (1)

$$
\begin{aligned}
& b_{1}=\boldsymbol{\Theta}_{1}^{T} \boldsymbol{\Xi}(\boldsymbol{x}) ; \boldsymbol{\Theta}_{1}=\left(\Theta_{11}, \Theta_{12}, \ldots, \Theta_{1 m}\right)^{T} \\
& b_{2}=\boldsymbol{\Theta}_{2}^{T} \boldsymbol{\Xi}(\boldsymbol{x}) ; \boldsymbol{\Theta}_{2}=\left(\Theta_{21}, \Theta_{22}, \ldots, \Theta_{2 m}\right)^{T} \\
& \ldots \\
& b_{n}=\boldsymbol{\Theta}_{n}^{T} \boldsymbol{\Xi}(\boldsymbol{x}) ; \boldsymbol{\Theta}_{n}=\left(\Theta_{n 1}, \Theta_{n 2}, \ldots, \Theta_{n m}\right)^{T}
\end{aligned}
$$




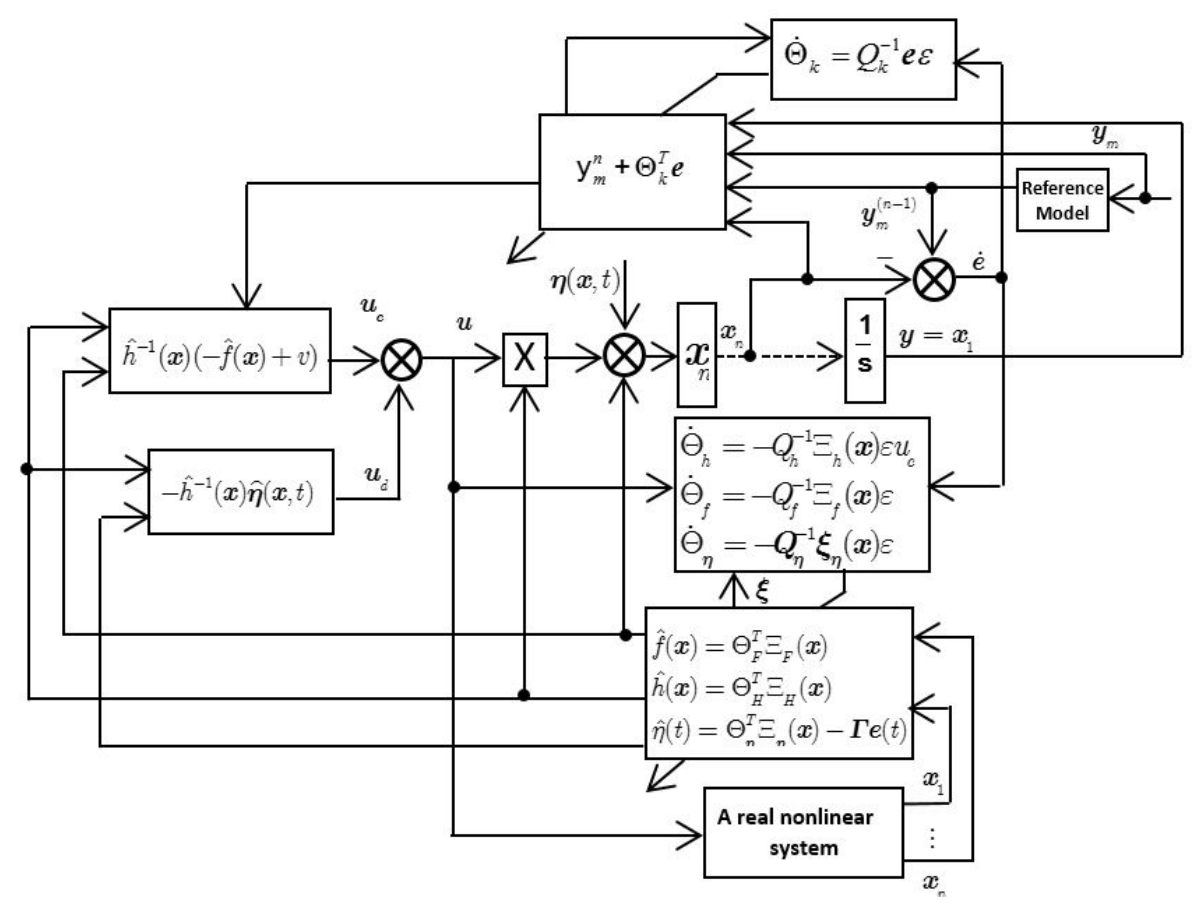

Figure 1. The scheme of new adaptive RBF neural network and disturbance estimation

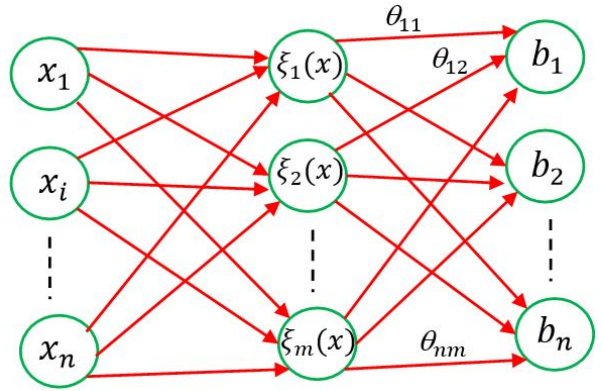

Figure 2. The structure of MIMO RBF neural network

This yields, $\boldsymbol{b}=\boldsymbol{\Theta} \Xi(\boldsymbol{x})$ where

$$
\begin{aligned}
& \boldsymbol{b}=\left(\begin{array}{c}
b_{1} \\
b_{2} \\
\vdots \\
b_{n}
\end{array}\right) \boldsymbol{\Theta}=\left(\begin{array}{cccc}
\Theta_{11} & \Theta_{12} & \cdots & \Theta_{1 m} \\
\Theta_{21} & \Theta_{22} & \cdots & \Theta_{2 m} \\
\vdots & \vdots & \ddots & \vdots \\
\Theta_{n 1} & \Theta_{n 2} & \cdots & \Theta_{n m}
\end{array}\right) \\
& \boldsymbol{\Xi}(\boldsymbol{x})=\left(\Xi_{1}(\boldsymbol{x}), \Xi_{2}(\boldsymbol{x}), . ., \Xi_{m}(\boldsymbol{x})\right)^{T}
\end{aligned}
$$

in which, the basis function $\Xi(x)$ is Gaussian function in (2). The unknown function $\boldsymbol{F}(\boldsymbol{x})$ and $\boldsymbol{H}(\boldsymbol{x}$ are approximated by RBF neural network (50) with $F(x)=$ $\boldsymbol{\Theta}_{F} \Xi_{F}(x)$ and $\hat{H}(x)=\boldsymbol{\Theta}_{\boldsymbol{H}} \Xi_{\boldsymbol{H}}(\boldsymbol{x})$. The $\boldsymbol{\Theta}_{\boldsymbol{H}}^{*}$ and $\boldsymbol{\Theta}_{\boldsymbol{F}}^{*}$ are called optimal values

$$
\boldsymbol{F}(\boldsymbol{x})=\Theta_{F}^{*} \Xi_{F}(x) ; H(x)=\Theta_{H}^{*} \Xi_{H}(x)
$$

and $\eta(x, t)=\boldsymbol{d}(x, t)+\Delta_{\boldsymbol{F}}(\boldsymbol{x})+\Delta_{\boldsymbol{H}}(\boldsymbol{x}) \boldsymbol{u}_{c}$ is lumped disturbance.

The proposed controller for MIMO nonlinear system (37)

$$
\begin{aligned}
& \boldsymbol{u}(t)=\boldsymbol{u}_{c}(t)+\boldsymbol{u}_{d}(t) \\
& \boldsymbol{u}_{c}=\hat{\boldsymbol{H}}^{-1}(\boldsymbol{x})(-\overline{\boldsymbol{x}} \boldsymbol{x}) \\
& \left.+\boldsymbol{y}_{m}^{(n)}+\boldsymbol{K}_{1} \boldsymbol{e}+\boldsymbol{K}_{2} \dot{\boldsymbol{e}}+\ldots+\boldsymbol{K}_{n-1} \boldsymbol{e}^{n-1}\right) \\
& \boldsymbol{u}_{d}=-\hat{\boldsymbol{H}}^{-1}(\boldsymbol{x}) \boldsymbol{\eta}(\boldsymbol{x}, t)
\end{aligned}
$$

in which $\boldsymbol{K}_{1}=\operatorname{diag}\left(k_{i 1}\right) ; \boldsymbol{K}_{2}=\operatorname{diag}\left(k_{i 2}\right) ; \ldots ; \boldsymbol{K}_{n-1}=$ $\operatorname{diag}\left(k_{i(n-1)}\right)$.

Theorem 3. The nonlinear system (37) with the controller (41) will be asymptotically stable. If the $\boldsymbol{\Theta}_{\boldsymbol{H}}, \boldsymbol{\Theta}_{\boldsymbol{F}}$ updated by the adaptive law

$$
\dot{\Theta}_{F}=-Q_{F}^{-1} \Xi_{F}(x) \varepsilon ; \dot{\Theta}_{H}=-Q_{H}^{-1} \Xi_{H}(x) \varepsilon u_{c}
$$

and the disturbance estimation is obtained form

$$
\hat{\eta}(x, t)=-\int_{0}^{t} \Xi_{\eta}(x)\left(Q_{\eta}^{-1}\right) \varepsilon d t \Xi_{\eta}(x)-\Gamma E(t)
$$

in which $\boldsymbol{E}=\left(\boldsymbol{e}, \dot{\boldsymbol{e}}, \ldots, \boldsymbol{e}^{n}\right)^{T}$ and $\boldsymbol{\Gamma}=\left(\gamma_{1}, \gamma_{2}, \ldots, \gamma_{n}\right) ; \gamma_{i}=$ $\operatorname{diag}\left(\gamma_{i j}\right) ; j=1,2, . ., n$ is root of

$$
\boldsymbol{\Gamma} \boldsymbol{A}_{D}+\boldsymbol{\Gamma} \boldsymbol{B}_{D} \boldsymbol{\Gamma}+\boldsymbol{\Xi}_{\eta}^{T}(\boldsymbol{x}) \boldsymbol{Q}_{\eta}^{-1} \boldsymbol{\Xi}_{\eta}(\boldsymbol{x}) \boldsymbol{a}_{D}=0
$$

Proof: Theorem 3 is proven similarly as the proof of Theorem 2. The adaptive law (42) can be found by using Lyapunov function

$$
V=\frac{1}{2} \varepsilon^{2}+\frac{1}{2} \tilde{\boldsymbol{\Theta}}_{F} \boldsymbol{Q}_{\boldsymbol{F}} \tilde{\boldsymbol{\Theta}}_{\boldsymbol{F}}^{T}+\frac{1}{2} \tilde{\mathbf{\Theta}}_{h} \boldsymbol{Q}_{\boldsymbol{H}} \tilde{\boldsymbol{\Theta}}_{\boldsymbol{H}}^{T}+\frac{1}{2} \tilde{\mathbf{\Theta}}_{\eta} \boldsymbol{Q}_{\eta} \tilde{\mathbf{\Theta}}_{\eta}^{T}
$$

and the dynamic error can be expressed

$$
\begin{aligned}
& \underbrace{\left(\begin{array}{c}
\dot{\boldsymbol{e}} \\
\vdots \\
\boldsymbol{e}^{(n-1)} \\
\boldsymbol{e}^{(n)}
\end{array}\right)}_{E}=\underbrace{\left(\begin{array}{c}
\boldsymbol{\Theta} \\
\vdots \\
\boldsymbol{\Theta} \\
\boldsymbol{I}
\end{array}\right)}_{\boldsymbol{B}_{\boldsymbol{D}}} e_{\eta}+ \\
& +\underbrace{\left(\begin{array}{cccc}
\boldsymbol{I} & \cdots & \Theta \\
\vdots & \vdots & \ddots & \vdots \\
\boldsymbol{\Theta} & \boldsymbol{\Theta} & \cdots & \boldsymbol{I} \\
-\boldsymbol{K}_{1} & -\boldsymbol{K}_{2} & \cdots & -\boldsymbol{K}_{n-1}
\end{array}\right)}_{A_{\boldsymbol{D}}}\left(\begin{array}{c}
\boldsymbol{e} \\
\vdots \\
\boldsymbol{e}^{(n-2)} \\
\boldsymbol{e}^{(n-1)}
\end{array}\right)
\end{aligned}
$$



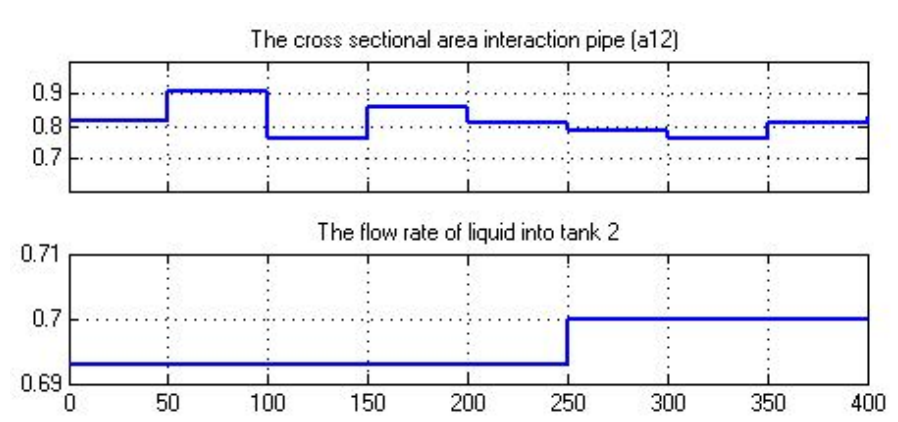

Figure 3. Two uncertain parameters of system

and notice that, in (44) $\boldsymbol{a}_{\boldsymbol{D}}=\left(\boldsymbol{K}_{1}, \boldsymbol{K}_{2}, \ldots, \boldsymbol{K}_{n}\right) \in$ $\boldsymbol{R}^{n \times(n \times n)} ; \boldsymbol{A}_{\boldsymbol{D}} \in \boldsymbol{R}^{(n \times n) \times(n \times n)} ; \boldsymbol{B}_{\boldsymbol{D}} \in \boldsymbol{R}^{(n \times n) \times n} ; \boldsymbol{\Gamma} \in$ $\boldsymbol{R}^{n \times(n \times n)} ; \boldsymbol{\Xi}(\boldsymbol{x}) \in \boldsymbol{R}^{1 \times m}$ and $\boldsymbol{Q}_{\eta} \in \boldsymbol{R}^{m \times m}$.

\section{ILlUSTRATIVE EXAMPLE}

\subsection{A coupled tank liquid level system}

In this section, we give an example of tracking reference set point with a coupled tank liquid level system. The simulation illustrates the convergence of error under our proposed TC-RBF and NA-RBF-DE approach. In [7], the modeled equation of coupled tank liquid level system and parameters given as

$$
\begin{aligned}
& \frac{d}{d t}\left(\begin{array}{l}
x_{1} \\
x_{2}
\end{array}\right)=\underbrace{\left(\frac{-b_{12} a_{12} \sqrt{2 g\left|x_{1}-x_{2}\right|}}{A_{1}}\right.}_{\boldsymbol{a}(\boldsymbol{x})})+ \\
& +\underbrace{\left(\begin{array}{c}
\frac{k}{A_{1}} \\
0
\end{array}\right)}_{\boldsymbol{b}(\boldsymbol{x})} u(t) ; y=x_{2}=g(\boldsymbol{x}) ; \boldsymbol{x}=\left(x_{1} x_{2}\right)^{T}=\left(h_{1} h_{2}\right)^{T}
\end{aligned}
$$

We use the Theorem [17] in order to convert (46) into (3) where

$$
f(x)=L_{a g}^{2} g(x) ; h(x)=L_{b} L_{a} g(x)
$$

We assume that the function of $\boldsymbol{f}(\boldsymbol{x})$ and $\boldsymbol{h}(\boldsymbol{x})$ describing the system dynamics are unknown (uncertain). The control target guarantees for the liquid level of Tank 2 at the set point. Opened valve ratio of pump 1 is adjusted by control law, and there are two uncertain parameters (the cross-sectional area interaction pipe $a_{12}$ and the flow rate of liquid into tank $2 a_{2}$ ) as Fig. 3. We would calculate the $\hat{f}(\boldsymbol{x}) ; \hat{h}(\boldsymbol{x}) ; \eta(t)$ by the adaptive RBF neural network which has 9 neurons in the hidden layer. The input vector of RBF is composed of two inputs $x=\left(x_{1} x_{2}\right)^{T}$, the radial basis functions are chosen as Gaussian functions as given:

$$
\phi_{b i}(\boldsymbol{x})=\exp \left(-\frac{\left(x_{1}-c_{1 i}\right)^{2}+\left(x_{2}-c_{2 i}\right)^{2}}{2 \sigma^{2}}\right)
$$

where the corresponding widths for every function is $\sigma=0.7$ and the centers of the basis function are evenly distributed in state space as Fig. 4 and $\Theta^{T}$ is connection weight matrix between the hidden layer and the output

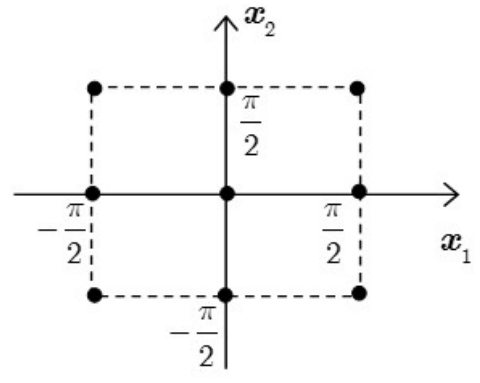

Figure 4 . The centers of basis function

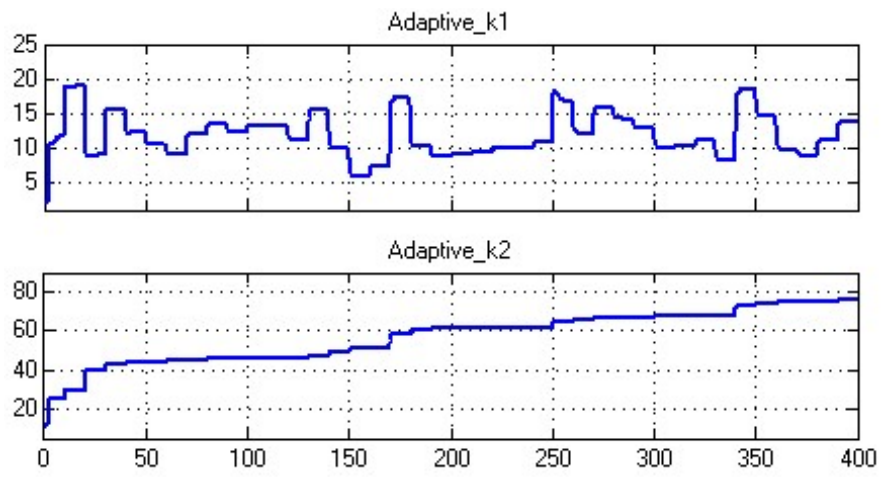

Figure 5. Adaptive gains of the NA-RBF-DE and TC-RBF

layer updated by adaptive law in Theorem 1 for TC-RBF and Theorem 2 for NA-RBF-DE. We choose the initial

Table I

THE PARAMETERS OF COUPLED LIQUID LEVEL SYSTEM

\begin{tabular}{cc} 
Parameter & Value \\
\hline$A_{1}, A_{2}$ the cross-sectional area of tank 1 and tank 2 & $100 \mathrm{~cm}^{2}$ \\
$Q_{\text {in }}$ the flow rate of liquid into tank 1 & $u\left(\mathrm{~cm}^{3} / \mathrm{sec}\right)$ \\
$Q_{\text {out }}$ the flow rate of liquid into tank 2 & $0.8 \mathrm{~cm}^{3} / \mathrm{sec}$ \\
$h_{1}, h_{2}$ the height of liquid in tank 1 and tank 2 & $\mathrm{~cm}^{2}$ \\
$b_{2}$ the cross sectional area of outlet pipe in tank 2 & $0.5 \mathrm{~cm}^{2}$ \\
$a_{12}$ the cross-sectional area interaction pipe & $\mathrm{cm}^{2}$ \\
$b_{12}$ the value ratio of interaction pipe & 1.5 \\
$g$ acceleration of gravity & $981 \mathrm{~cm}^{2} / \mathrm{sec}$ \\
$a_{2}$ the value ratio of outlet pipe of tank 2 & 1.5 \\
\hline
\end{tabular}

parameters $k_{p}(0)=1 ; k_{d}(0)=5$ of TC-RBF and NARBF-DE. In this coupled tank liquid level system, we consider that this system is affected by the disturbance $d(t)$ as Fig. 7. It notes that the labels of the horizontal axis of figures are the time and these of vertical axis of Fig. 6, 7 and Fig. 8 are the centimeter.

In the first case, we will compare our proposed methods TC-RBF to Adaptive fuzzy Sliding Mode Control (AFSMC) in [11]. Comparison of liquid level control simulation between TC-RBF and AFSMC is presented. A response of both TC-RBF and AFSMC are almost same. However, it is obviously that the tracking error for the TC-RBF due to reference liquid level is a much less than those by AFSMC see Fig. 6.

In second case, NA-RBF-DE is shown, the adaptive $\boldsymbol{\Theta}_{k}^{T}=\left(k_{1} k_{2}\right)$ of the NA-RBF-DE automatically alter when disturbance influence on system see Fig. 5. The disturbance estimator can be computed as Theorem 2 in 

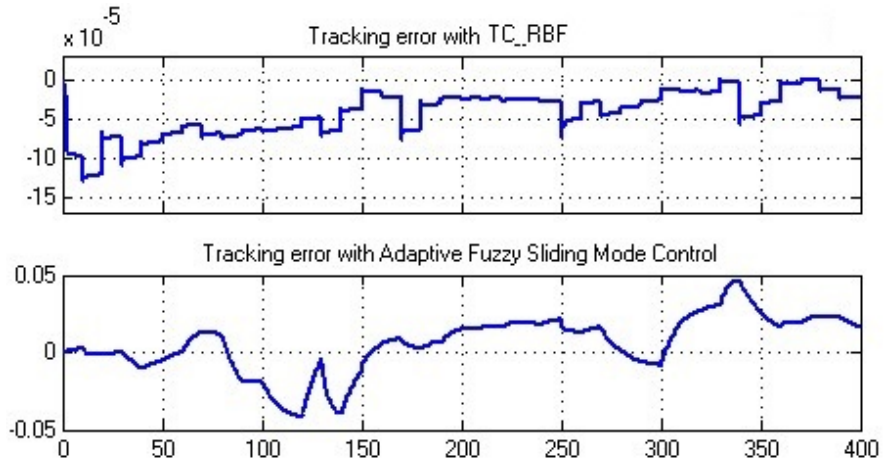

Figure 6. Tracking error with the disturbance of TC-RBF and AFSMC
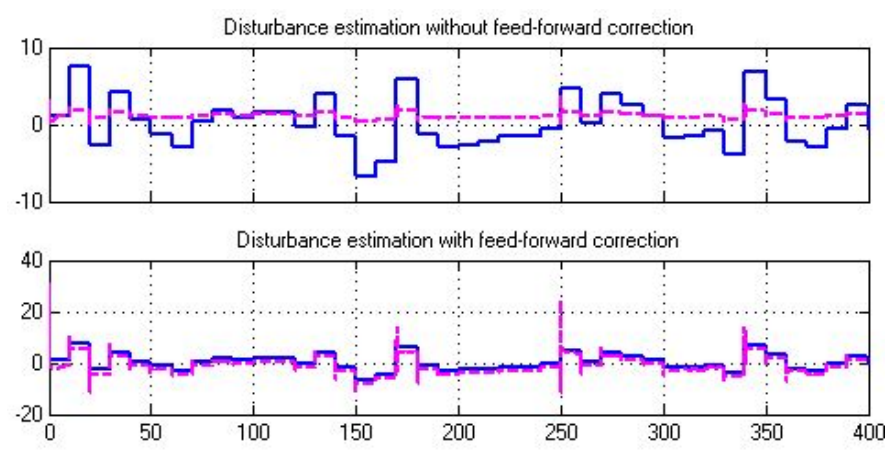

Figure 7. Disturbance estimation of TC-DE-RBF without $\gamma$ and with $\gamma$

which the feed-forward correction term $\gamma$ in (36) where:

$$
\begin{aligned}
& \boldsymbol{A}_{d}=\left(\begin{array}{cc}
0 & 1 \\
-k_{1} & -k_{2}
\end{array}\right) ; \boldsymbol{a}_{d}=\left(\begin{array}{ll}
-k_{1} & -k_{2}
\end{array}\right) ; \\
& \gamma=\left(\begin{array}{ll}
\gamma_{1} & \gamma_{2}
\end{array}\right) \\
& \boldsymbol{B}_{d}=\left(\begin{array}{ll}
0 & 1
\end{array}\right)^{T} ; \boldsymbol{Q}_{\eta}=0.01 \boldsymbol{I}_{(9 \times 9)} ; \boldsymbol{\Xi}_{\eta}(x)=\left(\begin{array}{l}
1 \mathbf{0}_{(1 \times 8)}
\end{array}\right) \\
& k_{1}(t)=k_{1}(0)+\frac{1}{q_{k 11}} \int\left(y_{m}-y\right) \varepsilon d t \\
& k_{2}(t)=k_{2}(0)+\frac{1}{q_{k 22}} \int\left(\dot{y}_{m}-\dot{y}\right) \varepsilon d t
\end{aligned}
$$

As mention, the lumped disturbance signal $\eta(x, t)$ contains both unknown input noise $d(x, t)$ and error structure $\delta_{f}(\boldsymbol{x}), \delta_{h}(\boldsymbol{x})$. The simulation results of the disturbed case are shown in Fig. 7, we can see that the disturbance estimator can be almost exactly disturbance term. It is clear that tracking error of NA-RBF-DE is very small, approximately $10^{-6}$ see Fig. 6 and Fig. 8 which is less than TC-RBF and is a lot less than AFSMC.
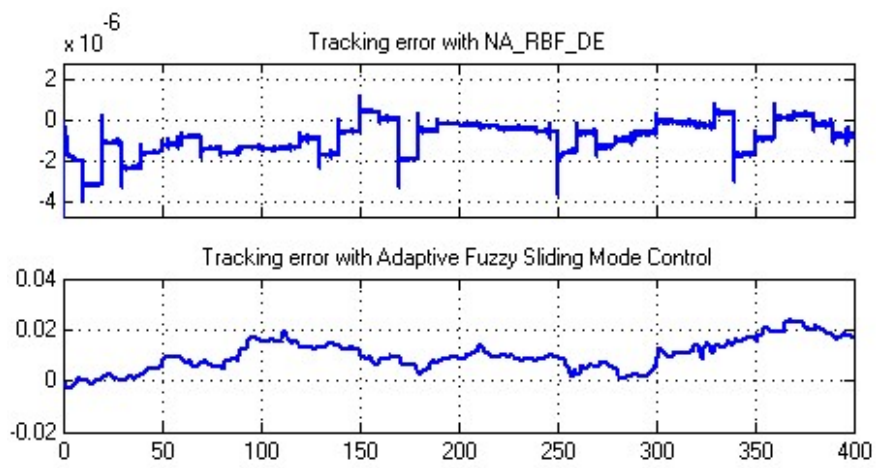

Figure 8. Tracking control with the disturbance of NA-RBF-DE and AFSMC

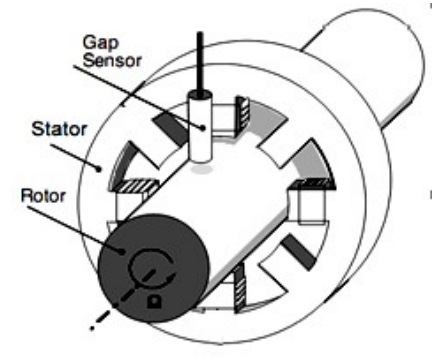

Figure 9. The two degree of freedom AMBs

\subsection{An active magnetic bearing system}

In this section, an active magnetic bearing system as Fig. 9 , used to illustrate our proposed methods. AMB system replaces mechanical bearings used in electric engine working in special environments [10], [18]. Because it uses magnetic forces to support the movement of the spindle without mechanical contacting, the new technology bearings have a number of advantages compared to other types of conventional bearings. However, this system is the unstable, so a feedback control loop is necessary to stabilize system. The control objective is that the position $x$ and $y$ of a suspended object follow

\begin{tabular}{|c|c|}
\hline Parameters & Values \\
\hline Mass of rotor $(k g)$ & $\mathrm{m}=12.4$ \\
\hline \multicolumn{2}{|l|}{ Displacements from the cylindrical rotor } \\
\hline center alignment to fixed origin $(m)$ & $h_{r t}=h=0.21$ \\
\hline Moment of inertia in k-axis $\left(\mathrm{kg} \cdot \mathrm{m}^{2}\right)$ & $J_{k}=6.88 * 10^{-3}$ \\
\hline \multicolumn{2}{|l|}{ Moment of inertia in $i$} \\
\hline and j-axis $\left(k g . m^{2}\right)$ & $J_{i}=J_{j}=2.22 * 10^{-1}$ \\
\hline Speed of rotor $(r p m)$ & 10000 \\
\hline The ratio electromagnetic-current $(N / A)$ & $K_{i}=102.325$ \\
\hline The ratio & \\
\hline electromagnetic-displacement $(N / A)$ & $K_{s}=4.65 * 10^{5}$ \\
\hline The gravity acceleration $\left(\mathrm{kg} . \mathrm{m} / \mathrm{s}^{2}\right)$ & $g=9.81$ \\
\hline
\end{tabular}
the position reference $x_{m}$ and $y_{m}$ with parameters of AMBs as Table II. The modeling a AMB in Euler

Table II

THE PARAMETERS OF AMBs

Lagrange equation follows as

$$
\boldsymbol{u}+\boldsymbol{d}(t)=\boldsymbol{D}(\boldsymbol{q}) \ddot{\boldsymbol{q}}+\boldsymbol{C}(\boldsymbol{q}, \dot{\boldsymbol{q}}) \dot{\boldsymbol{q}}+\boldsymbol{G}(\boldsymbol{q})
$$

where

$$
\begin{aligned}
& \text { where } \\
& \boldsymbol{D}(\boldsymbol{q})=\left(\begin{array}{cc}
\frac{2 J_{i}}{K_{i} l_{r t}^{2} \cos \theta_{x}} & 0 \\
0 & \frac{2 J_{i}}{K_{i} l_{l t r}^{2} \cos \theta_{y}}
\end{array}\right), \boldsymbol{G}(\boldsymbol{q})=\left(\begin{array}{c}
-\frac{m g h}{K_{i} l_{t t}^{2}} y \\
-\frac{m g h}{K_{i} l_{r t}^{2}} x
\end{array}\right) \\
& \boldsymbol{C}(\boldsymbol{q}, \dot{\boldsymbol{q}})=\left(\begin{array}{cc}
-\frac{J_{i} \sin \theta_{x}}{K_{i} l_{l t}^{3} \cos \theta_{x}} \dot{y} & -\frac{\omega_{r m} J_{k}}{K_{i} l_{t r}^{2} \cos \theta_{y}} \\
\frac{\omega_{r m} J_{k}}{K_{i} l_{r t}^{2} \cos \theta_{x}} & -\frac{J_{i} \sin \theta_{y}}{K_{i} l_{r t}^{3} \cos ^{3} \theta_{y}} \dot{x}
\end{array}\right), \boldsymbol{u}=\left(\begin{array}{c}
i_{y} \\
i_{x}
\end{array}\right)
\end{aligned}
$$

with $\theta_{x} ; \theta_{y}$ is very small, so we assume $\theta_{x} \approx \theta_{y}$, we obtain

$$
\begin{aligned}
\boldsymbol{D}(\boldsymbol{q}) & =\boldsymbol{D}(\boldsymbol{q})^{T}>0 ; \frac{d}{d t}\left(\frac{1}{\cos \theta_{x}}\right)=-\frac{\sin \theta_{x}}{l_{r t c} \cos ^{3} \theta_{x}} \dot{y} \\
\boldsymbol{D}(\boldsymbol{q}) & =\left(\begin{array}{cc}
\frac{-2 I_{i} \sin \theta_{x}}{K_{i} l_{r t} \cos ^{3} \theta_{x}} \dot{y} & 0 \\
0 & \frac{-2 J_{i} \sin \theta_{y}}{K_{K_{i}} l_{r t}^{3} \cos ^{3} \theta_{y}} \dot{x}
\end{array}\right) \\
& =\boldsymbol{C}(\boldsymbol{q}, \dot{\boldsymbol{q}})+\boldsymbol{C}^{T}(\boldsymbol{q}, \boldsymbol{q})
\end{aligned}
$$

and $\boldsymbol{q}=(x, y)^{T} \quad$ By introducing $\boldsymbol{x}=(\boldsymbol{q}, \dot{\boldsymbol{q}}, \ddot{\boldsymbol{q}})^{T}$ as state 


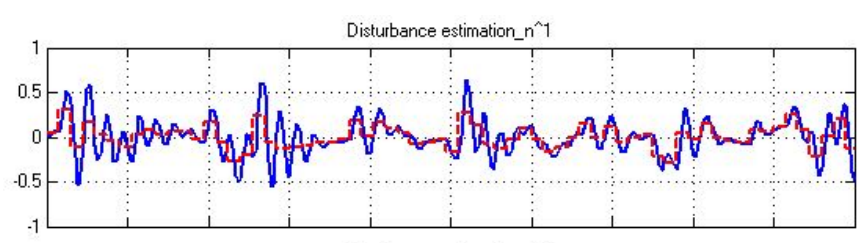

Disturbance estimation_n^2

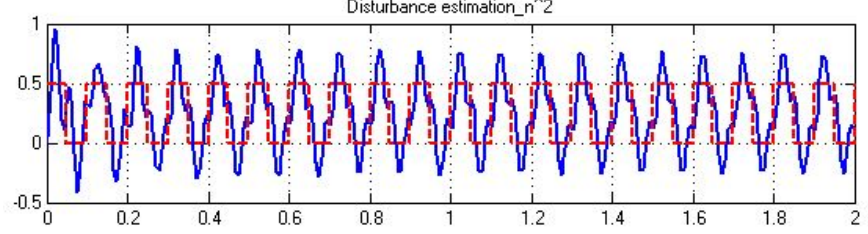

Figure 10. The disturbance estimation without the correction term $\Gamma$

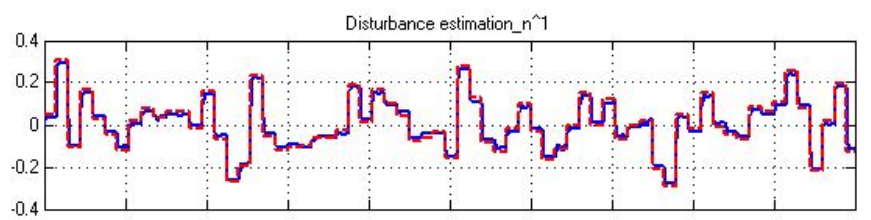

Disturbance estimation_n^2

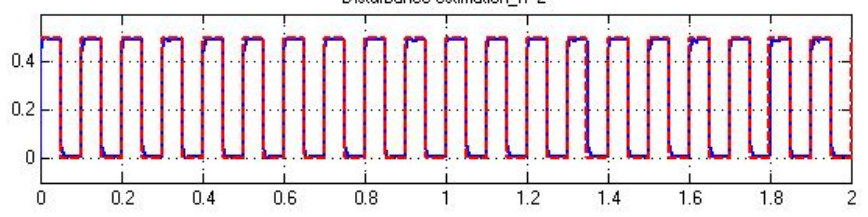

Figure 11. The disturbance estimation with the correction term $\Gamma$
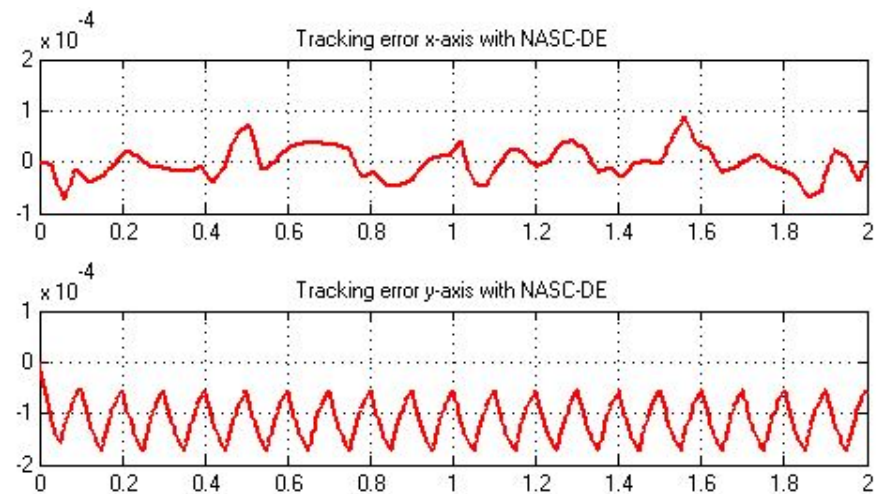

Figure 12. The tracking error of NASC-DE
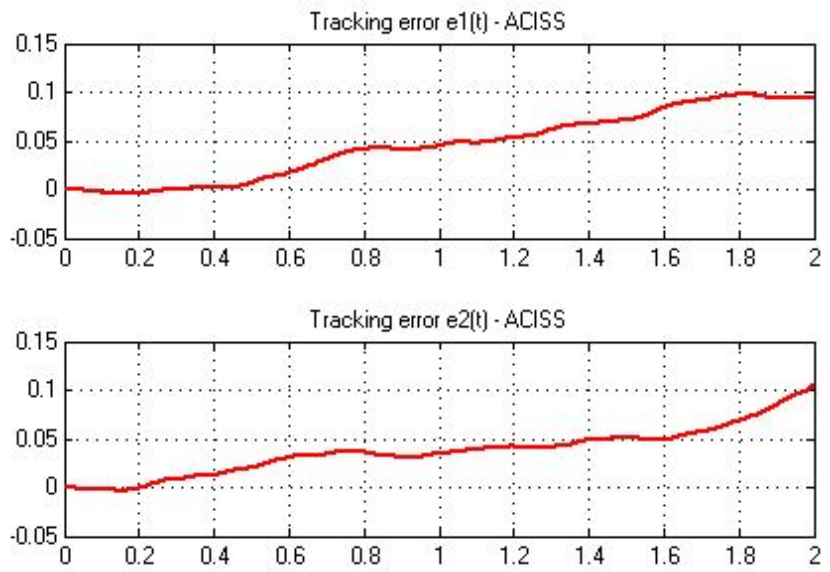

Figure 13. The tracking error of ACISS vector, equation (50) can be rewrite as

$$
\left\{\begin{array}{l}
\dot{x}_{1}=x_{2} \\
\dot{x}_{2}=\boldsymbol{F}(\boldsymbol{x})+\boldsymbol{H}(\boldsymbol{x}) \boldsymbol{u}+\boldsymbol{\eta}(\boldsymbol{x}, t) \\
\boldsymbol{y}=x_{1}
\end{array}\right.
$$

in which $\boldsymbol{F}(\boldsymbol{x})=\boldsymbol{D}^{-\mathbf{1}}(\boldsymbol{q})(\boldsymbol{C}(\boldsymbol{q}, \dot{\boldsymbol{q}}) \dot{\boldsymbol{q}}+\boldsymbol{G}(\boldsymbol{q})), \boldsymbol{H}(\boldsymbol{x})=$ $D^{-1}(q)$ and $\boldsymbol{\eta}(x, t)=D^{-1}(q) \boldsymbol{d}(t)$

We would calculate the $\overline{(x)} ; \hat{\boldsymbol{H}}(\boldsymbol{x}) ; \eta(t)$ by the adaptive RBF neural network which has 18 neurons in hidden layer. The input vector of RBF is composed of four inputs $x=(x \dot{x} ; y \dot{y})^{T}$, and the output vector of RBF has two outputs $\boldsymbol{b}=\left(b_{1} ; b_{2}\right)$ the radial basis functions are chosen as Gaussian functions as (48), the corresponding widths for every function is $\sigma=0.7$ and the centers of the basis function are evenly distributed in state space as Fig. 4. We would compare our proposed method NASC-DE to Adaptive Control based on ISS stabilization (ACISS) in [10]. We choose the fixed matrices $K_{1}, K_{2}$ of ACISS and NASC-DE

$$
\boldsymbol{K}_{1}=\left(\begin{array}{ll}
1 & 0 \\
0 & 1
\end{array}\right), \boldsymbol{K}_{2}=\left(\begin{array}{ll}
5 & 0 \\
0 & 5
\end{array}\right)
$$

Then, solving the equation (44) to find the feed-forward correction term $\Gamma$

$$
\begin{aligned}
& A_{D}=\left(\begin{array}{cc}
\boldsymbol{\Theta} & \boldsymbol{I} \\
-\boldsymbol{K}_{1} & -\boldsymbol{K}_{2}
\end{array}\right) ; \boldsymbol{a}_{D}=\left(\begin{array}{cc}
-\boldsymbol{K}_{1} & -\boldsymbol{K}_{2}
\end{array}\right) ; \\
& \boldsymbol{\Gamma}=\left(\begin{array}{llll}
\gamma_{1} & \gamma_{2} & \gamma_{3} & \gamma_{4}
\end{array}\right) \\
& \boldsymbol{B}_{D}=\left(\begin{array}{cc}
\boldsymbol{\Theta} & \boldsymbol{I}
\end{array}\right)^{T} ; \boldsymbol{Q}_{\eta}=0.01 \boldsymbol{I}_{(9 \times 9)} ; \boldsymbol{\Xi}_{\eta}(x)=\left(\begin{array}{ll}
1 & \mathbf{0}_{(1 \times 8)}
\end{array}\right)
\end{aligned}
$$

and the controller ACISS has form

$$
\boldsymbol{u}(t)=\boldsymbol{D}\left(\ddot{q}_{r}+\boldsymbol{K}_{1} \boldsymbol{e}+\boldsymbol{K}_{2} \dot{\boldsymbol{e}}-\boldsymbol{v}(t)\right)
$$

in which, a disturbance compensation signal $v(t)$ is chosen such as $|p(t)| \leq 0.5, \forall t$.

The simulations results of the case that a disturbance is considered are shown in Fig. 10 without feed-forward correction term $\Gamma$, the results of disturbance estimator with feed-forward correction term are almost as identical as real disturbance term (see Fig. 11). In addition, two kinds of disturbances are taken into account in this case. Based on this estimator, the disturbances are eliminated by the control law. This means that the performance of the tracking error of NASC-DE is very small (see Fig. 12), which is a much less than ACISS in Fig. 13. It is clear that the performance of close loop system is improved by using our proposed approaches. It notes that the labels of the horizontal axis of figures are the time and these of vertical axis of Fig. 10, 11, 12 and Fig. 13 are the centimeter.

\section{Conclusion}

The paper showed the disturbance estimator based on adaptive RBF neural network combined with the feedforward correction term, this method is applied for both uncertain nonlinear SISO and uncertain nonlinear MIMO system. Three proposed controllers (TC-RBF, NA-RBF-DE and NASC-DE), which combine disturbance estimation with adaptive parameter vector of the controller. Those approaches are applied for a class of 
nonlinear systems involving plan uncertainties and external disturbances containing SISO and MIMO system. The tracking performances are greatly improved by the use the disturbance estimator, adaptive RBF neural network and adaptive law of controller's parameters. We explore the effect of the controller from the simulation results.

\section{ACKNOWLEDGMENT}

This research was supported by the MOET (The Ministry of Education and Training), Viet Nam, under grant no. B2016-TN01-01.

\section{REFERENCES}

[1] A. Astolfi and L. Marconi, Analysis and Design of Nonlinear Control Systems. Springer Verlag, 2008.

[2] N. D. Phuoc, Analysis and control nonlinear System. Science and Technics Publishing House, Hanoi, 2012.

[3] T. V. Pham, L. K. Lai, Q. T. Nguyen, and M. T. Nguyen, "Disturbance estimation combined with new adaptive rbf neural network for uncertain system with disturbance," in Proceedings of the International Conference on Advanced Technologies for Communications (ATC). IEEE, 2016, pp. 247-252.

[4] M. Bahita and K. Belarbi, "Neural feedback linearization adaptive control for affine nonlinear systems based on neural network estimator," Serbian journal of electrical engineering, vol. 8, no. 3, pp. 307-323, 2011.

[5] G. Haigang, L. Hongxing, Z. Weijing, and S. Zhankui, "Direct adaptive fuzzy sliding mode control with variable universe fuzzy switching term for a class of mimo nonlinear systems," Mathematical Problems in Engineering, vol. 2012, 2012.

[6] S. Aloui, O. Pages, A. El Hajjaji, A. Chaari, and Y. Koubaa, "Robust adaptive fuzzy sliding mode control design for a class of mimo underactuated system," in Proceedings of the 18th IFAC World Congres, Milano (Italy), 2011.

[7] P. Van Thiem, L. K. Lai, and N. T. T. Quynh, "Improved adaptive fuzzy sliding mode control for second order nonlinear system," in Proceedings of the International Conference on Advances in Information and Communication Technology. Springer, 2016, pp. 255-264.

[8] Z. Bouchama, N. Essounbouli, M. Harmas, A. Hamzaoui, and K. Saoudi, "Reaching phase free adaptive fuzzy synergetic power system stabilizer," International Journal of Electrical Power E Energy Systems, vol. 77, pp. 43-49, 2016.

[9] Y. Tao, J. Zheng, and Y. Lin, "A sliding mode controlbased on a rbf neural network for deburring industry robotic systems," International Journal of Advanced Robotic Systems, vol. 13, no. 1, 2016.

[10] T. V. Pham and N. D. Phuoc, "Iss adaptive stabilization control for 2 degree of freedom active magnetic bearing modeling the system in euler-lagrange equation," Journal of Science and Technology Technical Universities, vol. 91, pp. 7-11, 2012.

[11] C. N. Van and P. N. Doan, "Adaptive tracking control based on disturbance attenuation and iss stabilization of euler-lagrange nonlinear systems in the presence of uncertainty and input noise," in Proceedings of the 2 nd International Conference on Artificial Intelligence, Management Science and Electronic Commerce (AIMSEC). IEEE, 2011, pp. 3698-3701.

[12] N. Manamanni, A. Hamzaoui, and N. Essounbouli, "Sliding mode control with adaptive fuzzy approximator for mimo uncertain systems," in Proceedings of the European Control Conference (ECC). IEEE, 2003, pp. 59-64.
[13] N. Essounbouli, A. Hamzaoui, and J. Zaytoon, “A supervisory robust adaptive fuzzy controller," in Proceedings of the 15th IFAC World Congres on Automatic and Control, vol. 35, no. 1. Elsevier, 2002, pp. 157-162.

[14] F. Qiao, Q. Zhu, A. F. Winfield, and C. Melhuish, “Adaptive sliding mode control for mimo nonlinear systems based on fuzzy logic scheme," International Journal of Automation and Computing, vol. 1, no. 1, pp. 51-62, 2004.

[15] A. Nasiri, S. K. Nguang, and A. Swain, "Adaptive sliding mode control for a class of mimo nonlinear systems with uncertainties," Journal of the Franklin Institute, vol. 351, no. 4, pp. 2048-2061, 2014.

[16] V. M. Popov and R. Georgescu, Hyperstability of control systems. Editura Academiei, Springer-Verlag, 1973.

[17] P. V. Thiem, L. K. Lai, and N. T. T. Quynh, "Tracking control of dynamic nonlinear systems via improved adaptive fuzzy control," International Journal of Engineering Research and Technology, vol. 5, no. 4, 2016.

[18] A. Chiba, T. Fukao, O. Ichikawa, M. Oshima, M. Takemoto, and D. G. Dorrell, Magnetic bearings and bearingless drives. Elsevier, 2005.

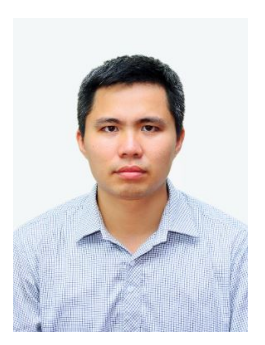

Thiem V. Pham received his B.Sc. in Automatic Control from Thai Nguyen University of Technology, and M.Sc. in Automatic Control from Ha Noi University of Science, Viet Nam, in 2009 and 2012, respectively. His current research interests include multi-agent systems, fault diagnosis, fault tolerant control and nonlinear control system. He is currently pursuing his Ph.D. study in Research Center of STIC (CReSTIC), University of Reims ChampagneArdenne (URCA), France.

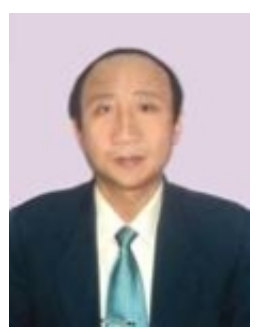

Lai K. Lai received the B.Eng. degree in Automatation Engineering from Thai Nguyen University of Technology in Vietnam in 1979. He received the M.Eng. degree in Automatic Control from Hanoi University of Technology in Vietnam, 1998. He received the Ph.D degree from Hanoi University of Technology in Vietnam in 2003. His main research areas are fuzzy logic control, neural network, model predictive control, power electronics and measuring methods. He is currently an associate professor in the Faculty of Electrical Institute of Thai Nguyen University ofTechnology, Thai Nguyen city, Vietnam. 